\title{
Accuracy of satellite orbit prediction and optical design of optical ground station beacons for satellite-to- ground optical communication
}

H. Takenaka, H. Kunimori, S. Fujita, T. Kuwahara, T. Kubooka, et al.

H. Takenaka, H. Kunimori, S. Fujita, T. Kuwahara, T. Kubooka, T. Fuse, M. Toyoshima, "Accuracy of satellite orbit prediction and optical design of optical ground station beacons for satellite-to-ground optical communication," Proc. SPIE 11852, International Conference on Space Optics — ICSO 2020, 118522C (11 June 2021); doi: 10.1117/12.2599372

SPIE Event: International Conference on Space Optics - ICSO 2021, 2021, Online Only 


\section{International Conference on Space Optics-ICSO 2020}

Virtual Conference

30 March-2 April 2021

Edited by Bruno Cugny, Zoran Sodnik, and Nikos Karafolas
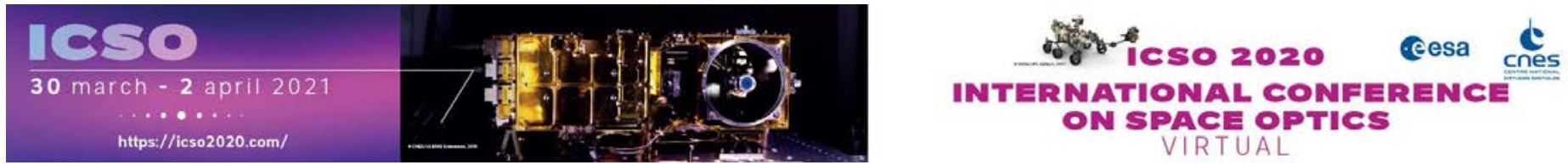

\section{Accuracy of satellite orbit prediction and optical design of optical ground station beacons for satellite-to-ground optical communication}

\section{- esa issporeatings denes}




\title{
Accuracy of satellite orbit prediction and optical design of optical ground station beacons for satellite-to-ground optical communication
}

\author{
H. Takenaka ${ }^{\mathrm{a} *}$, H. Kunimori ${ }^{\mathrm{a}}$, S. Fujita ${ }^{\mathrm{b}}$, T. Kuwahara ${ }^{\mathrm{b}}$, \\ T. Kubooka ${ }^{\mathrm{a}}$, T. Fuse ${ }^{\mathrm{a}}$, M. Toyoshima ${ }^{\mathrm{a}}$ \\ ${ }^{a}$ National Institute of Information and Communications Technology \\ Nukui-Kitamachi 4-2-1, Koganei, Tokyo 184-0014 Japan.; \\ b Tohoku University \\ 6-3, Aoba, Aramaki, Aoba-ku, Sendai 980-8578 Japan
}

\begin{abstract}
In recent years, spatial optical communication has been attracting attention as a large-capacity communication method that connects satellites and the ground. Radiofrequency communications are used as a method for transmitting data acquired by satellites to the ground. However, the radio frequencies used make it difficult to improve the communication speed, owing to interference problems and the carrier frequency. Space optical communication is expected to be a solution to this issue. A beacon method is often used to emit light from an optical ground station toward a satellite for initial acquisition. It is desirable that the divergence angle of the irradiating beacon covers the range of satellite orbit prediction. Therefore, it is important to know the orbit prediction accuracy of a satellite. In the experiment, satellite flare was observed from a ground station and verified by comparing the telescope tracking data with the GPS data and NORAD-TLE information mounted on the satellite.
\end{abstract}

Keywords: FSO, satellite orbit prediction, Two Line Element

\section{INTRODUCTION}

In recent years, the performance of observation equipment mounted on satellites has improved such that it can obtain an increased amount of data from only a single observation. Radiofrequency communications are used as a method for transmitting data acquired by satellites to the ground. However, the radio frequencies used make it difficult to improve the communication speed, owing to interference problems and the carrier frequency. Space optical communication is expected to be a solution to this issue.

In order to carry out satellite-to-ground optical communication, the method of irradiating the beacon light from the ground station to the satellite is often carried out. However, the angle of spread of the beacon light to the satellite depends on the accuracy of satellite orbit prediction, and a beacon light with a spread that covers the orbit error is ideal with a given limitation of required irradiance at a satellite.

A standard format for predicting satellite orbits is Two Line Element (TLE) information published by NORAD ${ }^{[1]}$. However, NORAD-TLE is updated infrequently and sometimes has a large error in order to establish optical communication links.

In this study, the orbit prediction is performed from GPS data acquired by the satellite, and the accuracy of the orbit prediction ${ }^{[2]}$ and NORAD-TLE is verified by comparing the accuracy of the GPS orbit prediction with that of the ground station tracking log and the GPS data during the tracking experiment using optical observations from the optical ground station. 


\section{SATELLITE AND OPTICAL GROUND STATION}

\subsection{RISESAT}

RISESAT is Rapid International Scientific Experiment Satellite, and an overview of RISESAT is shown in Table $1^{[3]}$. RISESAT has eight scientific missions and eight technology demonstration missions. One of them is an optical communication experiment using VSOTA ${ }^{[4]}$. RISESAT is a satellite with a size of $50 \mathrm{~cm}$ cube and equipped with a GPS receiver. In this experiment, the experiment was carried out using this GPS data.

Table 1. Overview of RISESAT

\begin{tabular}{|l|l|}
\hline \multicolumn{1}{|c|}{ Item } & \multicolumn{1}{c|}{ Value } \\
\hline Size & $50 \mathrm{~cm} \times 50 \mathrm{~cm} \times 50 \mathrm{~cm}$ \\
\hline Weight & $60 \mathrm{~kg}$ \\
\hline Orbit & Sun Synchronous, Altitude: $500 \mathrm{~km}$ \\
\hline Attitude Control & Method: 3-axis stabilization \\
\hline Sampling rate of GPS-log & Variable \\
\hline
\end{tabular}

\subsection{Optical ground station (OGS)}

Figure 1 shows an overview of the optical ground station. The OGS is a Cassegrain reflecting telescope with a $1 \mathrm{~m}$ aperture. Table 2 shows the performance of OGS. The tracking performance of the LEO satellite is less than 10 seconds accurate. This telescope system has one Cassegrain focus, two Nathmys focal points, and two Coude focal points. The azimuth and elevation tracking logs of the telescope are recorded at $10 \mathrm{~Hz}$.

Table 3 shows the specifications of the cameras used in the experiment. In this experiment, this camera was installed at the Cassegrain focal point of the optical ground station, and the image of the satellite reflected by sunlight was acquired by the camera. The experiment was carried out with the integration time of the camera set to 1 second. The telescope and camera are calibrated using stars. However, even if star-calibration is performed, all errors cannot be removed, so the residual error is approximately 0.005 degrees.

Table 2. Overview of OGS ${ }^{[5]}$

\begin{tabular}{|l|l|}
\hline \multicolumn{1}{|c|}{ Item } & \multicolumn{1}{c|}{ Value } \\
\hline Optics type & Classical Cassegrain reflector \\
\hline Type of mount & Altitude-Azimuth drive mount \\
\hline Effective aperture of the primary mirror & $1000 \mathrm{~mm}$ \\
\hline Cassegrain focal ratio & $\mathrm{F}=12$ \\
\hline Synthetic focal length & $12000 \mathrm{~mm}$ \\
\hline Tracking accuracy & 10 arcsec accuracy for LEO satellite \\
\hline Pointing accuracy & 3 arcsec for celestial object higher than 30 deg \\
\hline Encoder & Optical incremental encoder \\
\hline Sampling rate of telescope-log & $10 \mathrm{~Hz}$ \\
\hline
\end{tabular}

Table 2. Camera performance

\begin{tabular}{|l|l|}
\hline \multicolumn{1}{|c|}{ Item } & \multicolumn{1}{c|}{ Value } \\
\hline Video output format & Monochrome \\
\hline Camera pixel size (width $\times$ height) & $5496 \mathrm{px} \times 3672 \mathrm{px}$ \\
\hline field of view (width $\mathrm{x}$ height) & $0.0636 \mathrm{deg} \times 0.0416 \mathrm{deg}$ \\
\hline
\end{tabular}




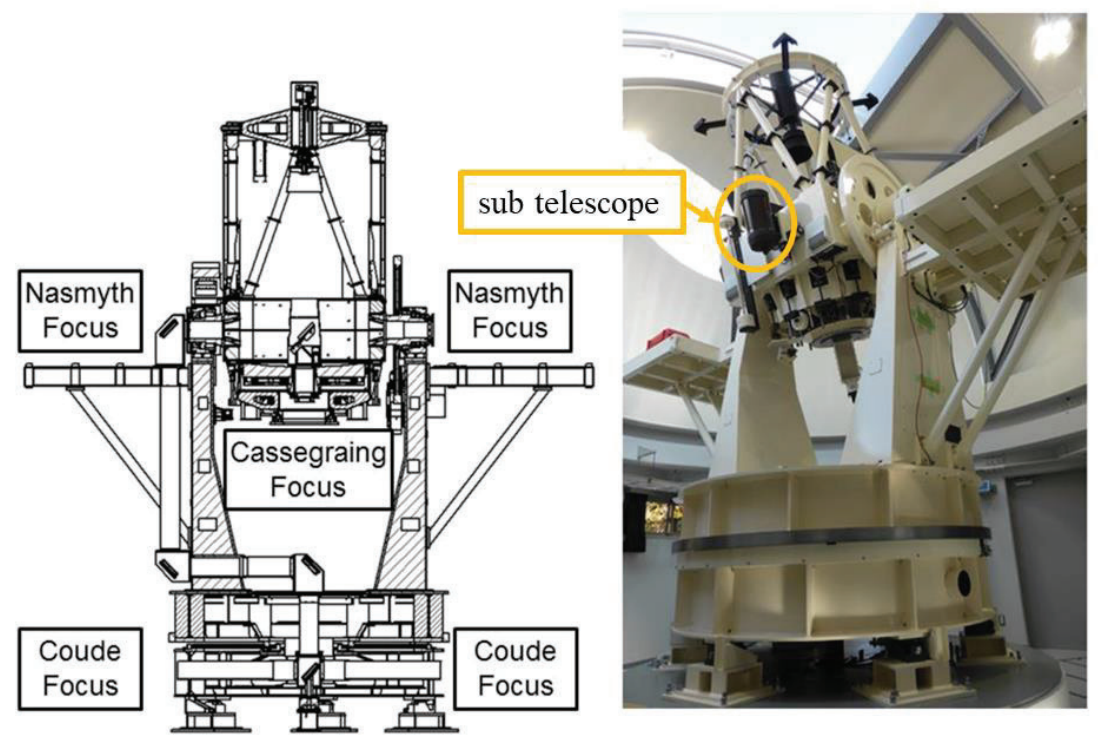

Figure 1. Overview of RISESAT

\section{MEASUREMENT EXPERIMENT}

The telescope tracked the satellite using TLE information and observed the satellite from OGS using the satellite flare. Figure 2 shows the Azimuth and Elevation of OGS at the time of the experiment. Also, the time that the satellite flare could be measured was about 160 seconds. Figure 3 shows an example of the camera image acquired during the experiment.

In the experiment, the satellite flare may not be within the field of view of the camera installed in Cassegrain-focus. Therefore, the satellite flare is first captured using the sub-telescope with a wider field of view shown in Figure 1. After that, the telescope was given an angle offset so that the satellite flare could be captured by the camera installed in Cassegrain-focus.
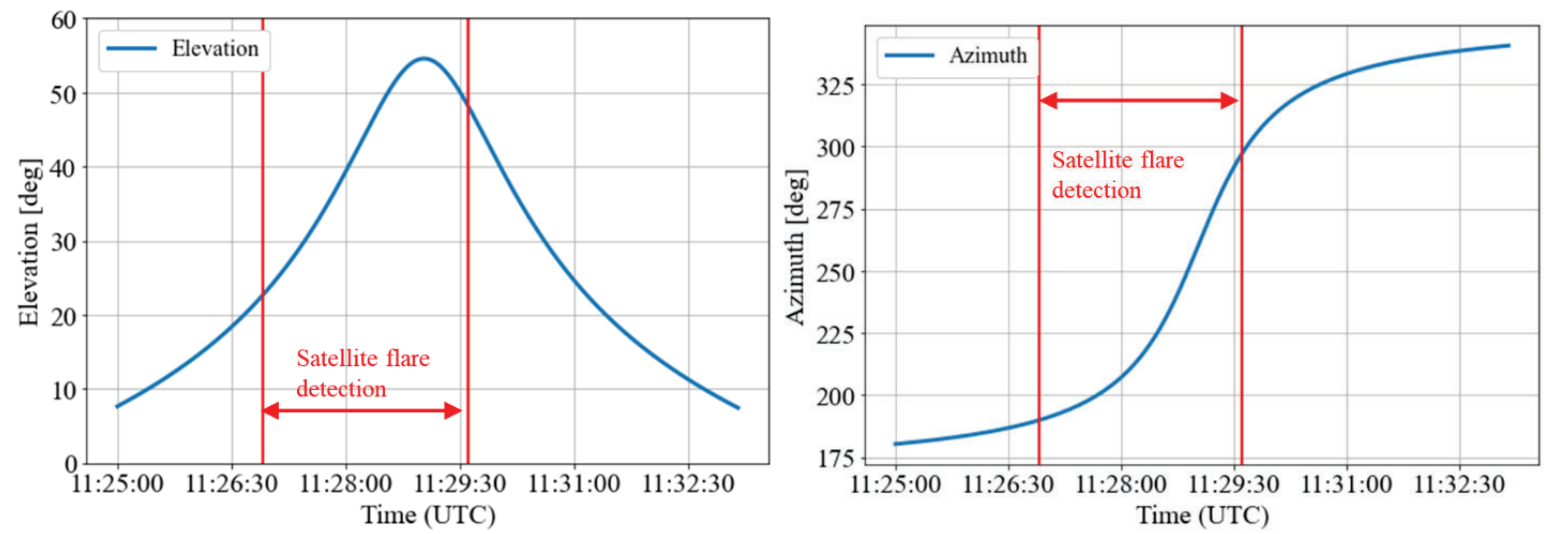

Figure 2. Azimuth and Elevation of OGS and satellite flare detection time 


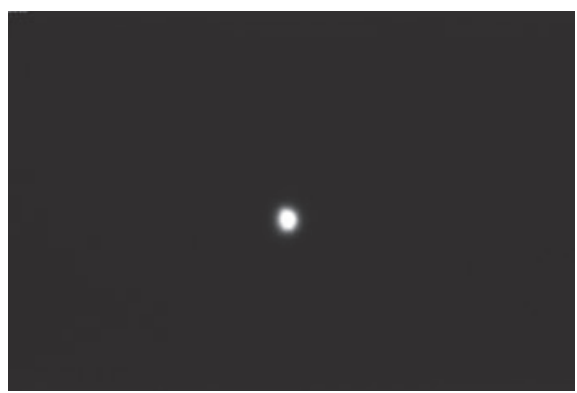

Figure 3. An example of a satellite flare image taken by the OGS camera

\section{EXPERIMENTAL DATA ANALYSIS}

\subsection{Processing of camera data}

The satellite position in the camera is calculated from the camera acquisition data from the brightness center of gravity. However, the amount of light received by the camera fluctuates significantly due to the tilt of the satellite, the influence of clouds, background noise, etc., in the reflection of sunlight on the satellite. Therefore, if the threshold value is easily obtained and the brightness center of gravity is obtained, an error increase. Therefore, we performed image processing according to the procedure in Table 3 . The result of the image processing is shown in Figure 4.

This image processing obtained the brightness center of gravity, and the tracking error of the telescope was corrected from the camera viewing angle and the number of pixels in Table 2. Figure 5 shows the amount of correction obtained from the image. The amount of correction in this experiment was in the range of -0.03 degrees to +0.03 degrees.

Table 3. Order of Image processing

\begin{tabular}{|c|l|}
\hline Step & \multicolumn{1}{|c|}{ Filter name } \\
\hline 1 & Binarization filter by threshold \\
\hline 2 & Convolution filter \\
\hline 3 & Erosion filter \\
\hline 4 & Binarization filter by threshold \\
\hline 5 & Dilation filter \\
\hline 6 & Erosion filter \\
\hline
\end{tabular}

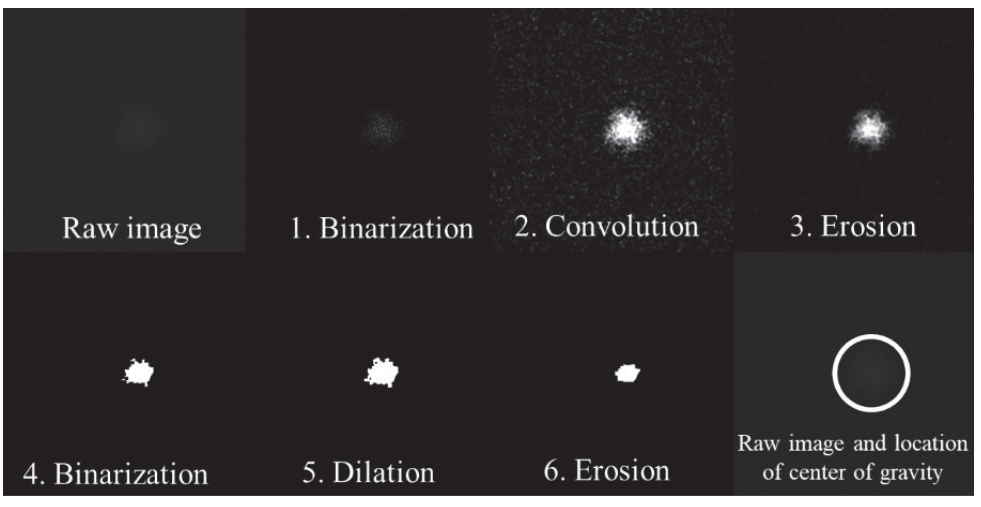

Figure 4. An example of Image filter application result (Enlarged view) 


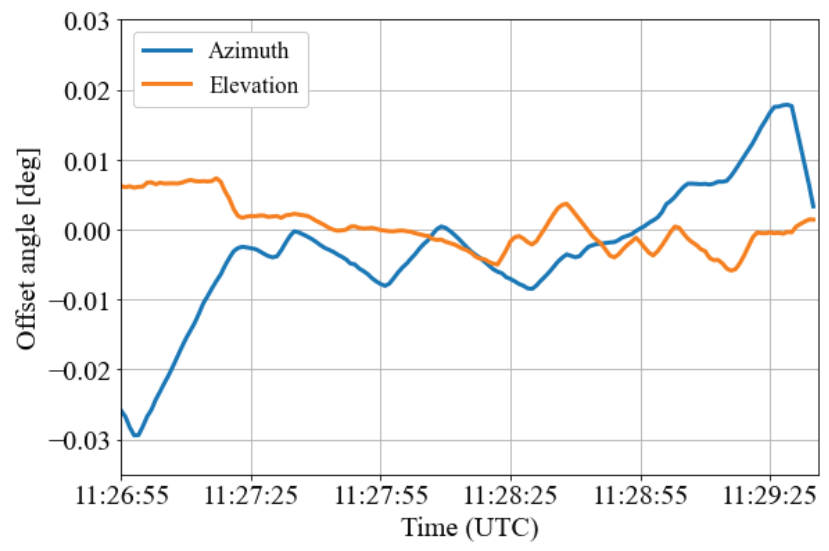

Figure 5. Telescope offset angle obtained from camera images

\subsection{Comparison of predicted and telescope tracking data}

The telescope tracking data corrected from the camera image, satellite GPS data, and TLE information were compared. We used the following TLEs, NORAD TLE epoch time about 8 hours before the experiment (NORAD-TLE (before 8h)), NORAD TLE about 0.5 hours after the experiment (NORAD-TLE (after $0.5 \mathrm{~h}$ )), and TLE generated from GPS data of RISESAT. The TLE generated from the GPS data of RISESAT was generated using GPS data sampled every 20 seconds from about 9 hours to 14 hours before the experiment time ${ }^{[2]}$.

Figure 6 shows the results of comparing each data based on the telescope tracking data corrected from the camera image. Figure 7 shows the total error amount of azimuth and elevation angle using Equation 1. $o_{a z}$ and $o_{e l}$ is azimuth and elevation angle of telescope tracking, $t_{a z}$ and $t_{e l}$ is of azimuth and elevation angle of each data to be compared.

$$
E_{r}=\sqrt{\left(o_{a z}-t_{a z}\right)^{2}+\left(o_{e l}-t_{e l}\right)^{2}}
$$
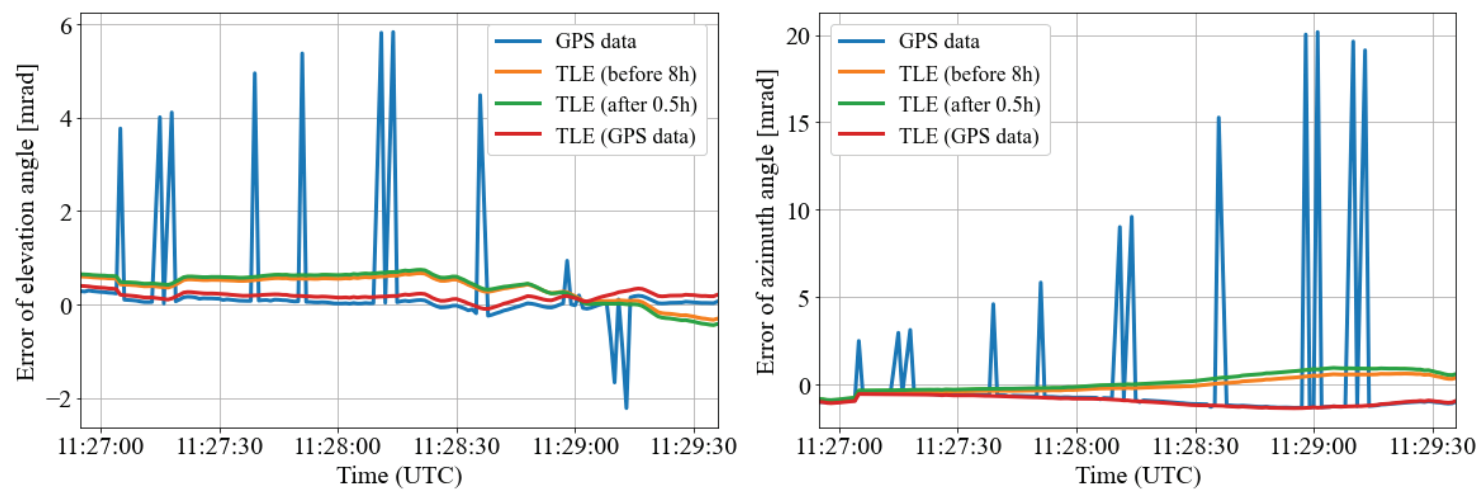

Figure 6. Compare each data based on telescope tracking data corrected from camera images

Tracking errors of GPS data sometimes show a value that is largely deviated due to GPS data errors. The error of the TLE generated from the RISESAT GPS data was the smallest in the elevation tracking error, and the NORAD-TLE (before 8h) was the smallest in the azimuth tracking error. The sizeable initial fluctuation in the azimuth angle is due to the large offset of the telescope in the azimuth angle direction to capture the satellite at the center of the camera. 
The best result was shown when tracking with the NORAD-TLE (before $8 \mathrm{~h}$ ) in the evaluation of the elevation angle and the azimuth angle in figure 7. It was found that the fluctuation of the elevation angle error was small as a whole, and the fluctuation of the azimuth angle error was larger in the analysis of this experiment.

According to the results of this experiment, it is considered that an uplink beacon with a divergence angle of 1 milliradian can be captured at a low elevation angle. As the elevation angle increases, the azimuth error fluctuates largely. Therefore, it is necessary to prepare an uplink beacon with a wider divergence angle or move the beam such as a spiral scan, when considering the entire experiment time. Since the satellite's position error tends to be primarily deviated by the azimuth angle, it seems good to give an offset in the azimuth angle direction to adjust the uplink beacon.

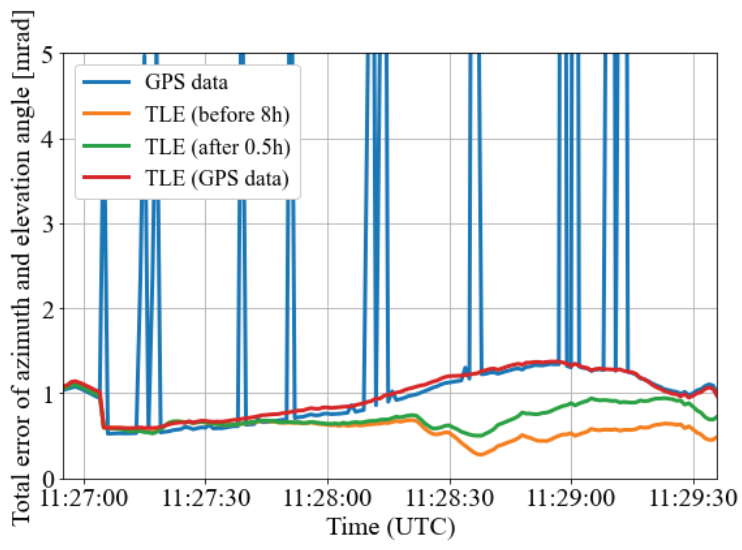

Figure 7. Total error of azimuth and elevation angle

\section{CONCLUSION}

In this paper, the telescope tracking data corrected from the camera image, satellite GPS data, and TLE information were compared. Comparing the telescope tracking data with each data, NORAD-TLE of epoch time about 8 hours before the experiment showed lower errors in this experiment measurement range. It was found that the fluctuation of the elevation angle error was small as a whole, and the fluctuation of the azimuth angle error was larger in the analysis of this experiment. This experimental case may have been exceptional, so I would like to continue experimenting and accumulating data.

\section{ACKNOWLEDGEMENTS}

This research and development work was supported by the MIC/SCOPE \# 191502001.

\section{REFERENCES}

[1] T.S. Kelso, "CelesTrak," <https://celestrak.com/> (19 October 1997)

[2] B. T. Honda, T. Kuwahara, S. Fujita, A. A. Pala, and Y. Shibuya, "High Precision Orbit Determination Method Based on GPS Flight Data for ALE-1,” 32nd Int. Symp. Sp. Technol. Sci. Nano Satell. Symp., vol. 1, pp. 1-8, 2019.

[3] Kuwahara, T., Yoshida, K., Sakamoto, Y., Tomioka, Y., Fukuda, K., and Fukuyama, M., "International Scientific Micro-satellite RISESAT based on Space Plug and Play Avionics," 26th Annual AIAA/USU Conference on Small Satellites, 2012.

[4] H. Takenaka, H. Kunimori, T. Kuwahara, Y. Sakamoto, S. Fujita, H. Tomio, M. Sakal, et al., "Optical communication experiment with microsatellite body-pointing using VSOTA on RISESAT," in ICSSC, 2019 , pp. $1-4$.

[5] M. Akioka, H. Takenaka, M. Toyoshima, Y. Koyama, Y. Takayama, and T. Seki, “The NICT's New OGS for Satellite Laser Communication and SOTA/SOCRATES experiment," in SpaceOps Conferences, 2016, pp. 1-6. 